\title{
THE ROSAT X-RAY SPECTRA OF BL LACERTAE OBJECTS
}

\author{
PAOLO PADOVANI \\ Dipartimento di Fisica, II Università di Roma "Tor Vergata" \\ Via della Ricerca Scientifica 1, I-00133 Roma, Italy \\ AND \\ PAOLO GIOMMI \\ SAX, Scientific Data Center, ASI \\ Viale Regina Margherita 202, I-00198 Roma, Italy
}

\section{Introduction}

We have analyzed the X-ray spectra of all BL Lacs observed (as pointed or serendipitous sources) by ROSAT. Spectral indices were obtained from the hardness ratios given in the WGA catalogue [5], a large list of X-ray sources generated from all the ROSAT PSPC pointed observations. The selection of the objects was done by cross-correlating the first revision of the WGA catalogue with our recent catalogue of BL Lacs ([3]). This resulted in 163 observations of 85 distinct BL Lacs, which correspond to about half the confirmed BL Lacs presently known. This represents the largest number of $B L$ Lacs for which homogeneous $\mathrm{X}$-ray spectral information is available and the largest BL Lac sample ever studied at $X$-ray frequencies.

Following our previous work $([1],[2])$, we divide the BL Lacs into HBLs (high-energy cutoff BL Lacs) and LBLs (low-energy cutoff BL Lacs) on the basis of their X-ray-to-radio flux ratios $\left(f_{\mathrm{X}} / f_{\mathrm{r}} \sim 10^{-11.5}\right.$ being the dividing line), and look for any differences in the X-ray spectra of the two classes. A full presentation of these results will be given elsewhere ([4]).

\section{Main Results}

BL Lacs have energy power-law spectral indices between 0 and 3 with a mean value $\alpha_{\mathrm{x}} \sim 1.4\left(f_{\nu} \propto \nu^{-\alpha}\right)$. Significant differences, however, are present between HBLs (generally selected in the X-ray band: 58 objects), and LBLs (generally found in radio surveys: 27 objects), with $\left\langle\alpha_{\mathrm{x}, \mathrm{HBL}}\right\rangle=$ 


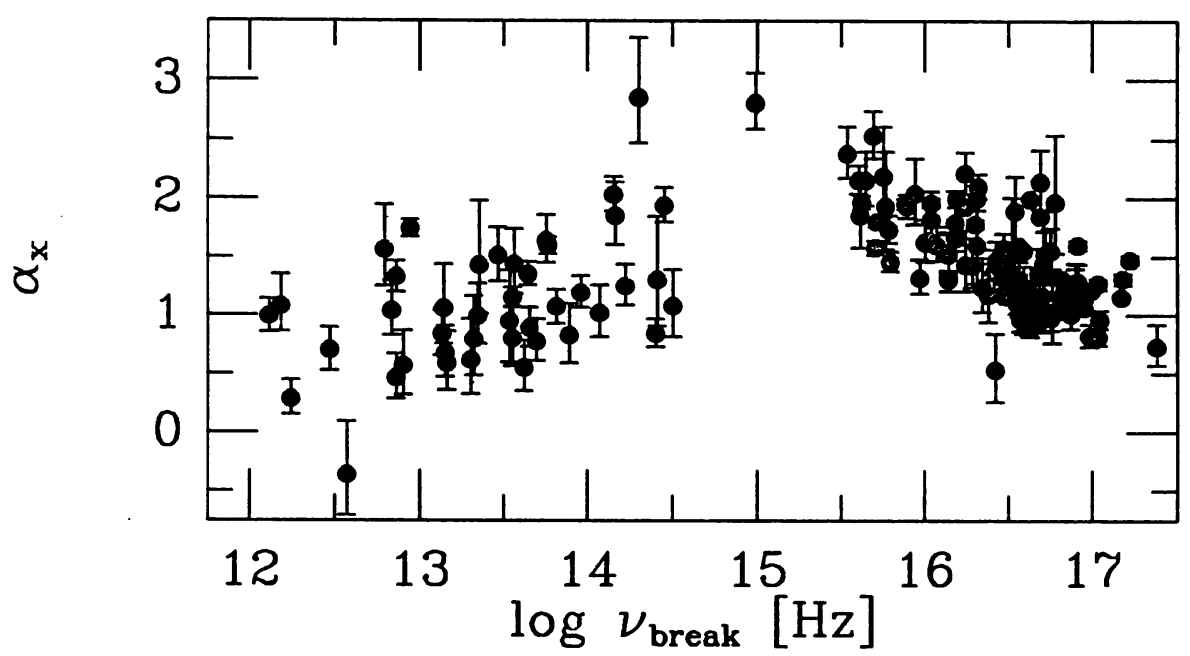

Figure 1. The X-ray spectral index versus the logarithm of the break frequency for $\mathrm{BL}$ Lacs. Objects with $\nu_{\text {break }} \gtrsim 10^{15} \mathrm{~Hz}$ have $f_{\mathrm{x}} / f_{\mathrm{r}}>10^{-11.5}$ and are, by definition, HBLs.

$1.52 \pm 0.06$ and $\left\langle\alpha_{x, \text { LBL }}\right\rangle=1.06 \pm 0.09$, different at the $99.99 \%$ level according to a Student's t-test. Moreover, the X-ray spectral indices of HBLs anticorrelate strongly $\left(99.99 \%\right.$ level) with $f_{\mathrm{x}} / f_{\mathrm{r}}$. No correlation is present for LBLs. Similarly, a strong correlation $\left(99.99 \%\right.$ level) exists between $\alpha_{x}$ and $\alpha_{\text {ox }}$ for HBLs but not for LBLs. The two classes separate extremely well on the $\alpha_{\mathrm{ox}}-\alpha_{\mathrm{x}}$ plane, that is all HBLs apart from one have $\alpha_{\mathrm{x}} \geq \alpha_{\mathrm{ox}}$ (within the errors), while the majority of LBLs have $\alpha_{\mathrm{x}}<\alpha_{\mathrm{Ox}}$. The most interesting correlation is, we believe, that between $\alpha_{\mathrm{x}}$ and the frequency of the energy cutoff, $\nu_{\text {break }}$, which we have estimated from the location of the objects on the $\alpha_{\mathrm{ro}}-\alpha_{\mathrm{ox}}$ plane ([2]). A strong anti-correlation (significant at the $99.99 \%$ level) is present between $\mathrm{X}$-ray spectral index and break frequency for HBLs, while LBLs display a weaker, positive, correlation (see Figure 1).

We interpret these results in terms of different mechanisms being responsible for the $\mathrm{X}$-ray emission in the two classes, i.e., synchrotron and inverse Compton for HBLs and LBLs respectively. The observed differences are consistent with the hypothesis that HBLs and LBLs are powered by the same non-thermal engines with different synchrotron cutoff energies.

\section{References}

[1] Giommi, P. and Padovani, P., 1994, MNRAS, 268, L51 310, 325

[2] Padovani, P. and Giommi, P., 1995a, ApJ, 444, 567

[3] Padovani, P. and Giommi, P., 1995b; MNRAS, in press

[4] Padovani, P. and Giommi, P., 1995c, MNRAS, submitted

[5] White, N.E., Giommi, P. and Angelini, L., 1994, IAUC 6100 